\title{
Application of Kalman Filter to Remove TMS-Induced Artifacts from EEG Recordings
}

\author{
Fabio Morbidi, Student Member, IEEE, Andrea Garulli, Senior Member, IEEE, \\ Domenico Prattichizzo, Member, IEEE, Cristiano Rizzo, and Simone Rossi
}

\begin{abstract}
Transcranial magnetic stimulation (TMS) is a technique in which a pulsed magnetic field created by a coil positioned next to the scalp is used to locally depolarize neurons in brain cortex. TMS can be combined with electroencephalography (EEG) to visualize regional brain activity in response to direct cortical stimulation, making it a promising tool for studying brain function. A technical drawback of EEG/TMS coregistrations is that the TMS impulse generates high amplitude and long-lasting artifacts that corrupt the EEG trace. In this brief, an offline Kalman filter approach to remove TMS-induced artifacts from EEG recordings is proposed. The Kalman filter is applied to the linear system arising from the combination of the dynamic models describing EEG and TMS signals generation. Time-varying covariance matrices suitably tuned on the physical parameters of the problem allow us to model the non-stationary components of the EEG/TMS signal, (neglected by conventional stationary filters). Experimental results show that the proposed approach guarantees an efficient deletion of TMS-induced artifacts while preserving the integrity of EEG signals around TMS impulses.
\end{abstract}

\section{INTRODUCTION}

$\mathbf{T}$ HANKS to some recent technological advances, neuroscience became one of the leading research areas, attracting scientists not only from the medical area but also from engineering, physics, biology, and psychology. Today, a large number of problems in neuroscience could benefit of tools commonly exploited in these different fields. Electroencephalographic signals processing, for instance, revealed an appealing test bed for filtering strategies developed in the control engineering area (see, e.g., [2], [5], [6], [15], [25], just restricting to the literature dealing with Kalman filtering).

Electroencephalography (EEG) is the neurophysiologic measurement of brain electrical activity recorded by electrodes placed on the scalp. EEG is frequently used in experimentation because the process is noninvasive to the research subject who does not need to make any specific action during the recording. A peculiar feature of EEG over other existing techniques is its excellent temporal resolution: in fact changes in brain electrical activity with a time scale of milliseconds can be easily detected.

Manuscript received July 27, 2007; revised December 16, 2007. Manuscript received in final form February 14, 2008. First published June 13, 2008; current version published October 22, 2008. Recommended by Associate Editor F. Caccavale.

F. Morbidi, A. Garulli, and D. Prattichizzo are with the Dipartimento di Ingegneria dell'Informazione, Università di Siena, 53100 Siena, Italy (e-mail: morbidi@dii.unisi.it; garulli@dii.unisi.it; prattichizzo@dii.unisi.it).

C. Rizzo is with the Micromed s.r.l., 31021 Mogliano Veneto (TV), Italy (e-mail: cristiano.rizzo@micromed-it.com).

S. Rossi is with the Dipartimento di Neuroscienze, Sezione Neurologia, Università di Siena, Policlinico "Le Scotte", 53100 Siena, Italy (e-mail: rossisimo@unisi.it).

Color versions of one or more of the figures in this paper are available online at http://ieeexplore.ieee.org.

Digital Object Identifier 10.1109/TCST.2008.921814

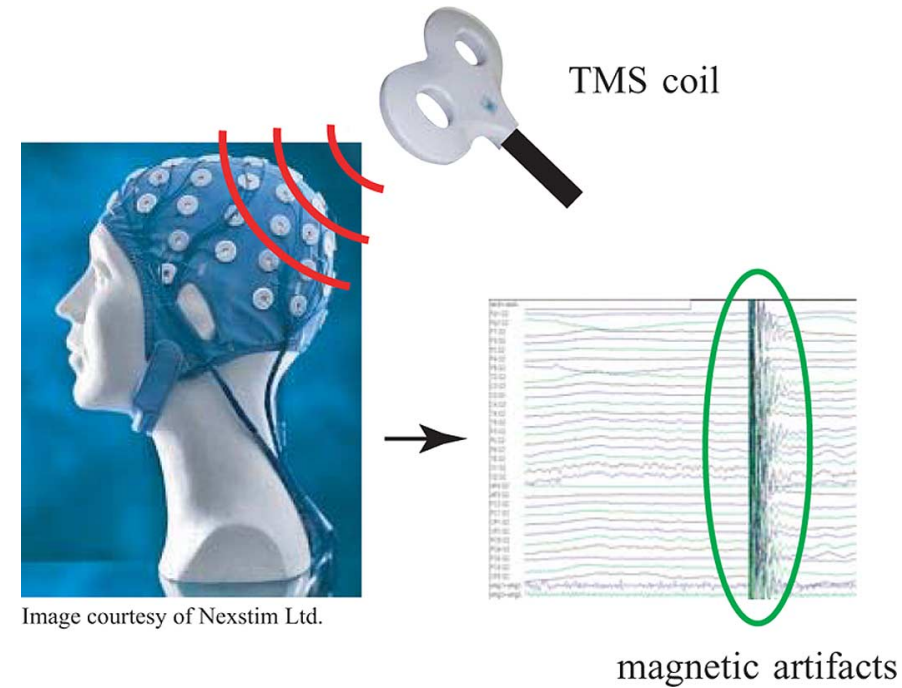

Fig. 1. TMS induces high amplitude artifacts on the EEG trace.

Recently a new powerful tool to investigate brain function has been introduced in neuroscience: the transcranial magnetic stimulation (TMS). TMS allows noninvasive brain stimulation using rapidly time-varying magnetic fields generated by a coil positioned in contact with the scalp [7], [13]. Besides the investigation of corticospinal motor pathways after stimulation of the motor cortex [13], [23], TMS of non-motor areas offered the unique opportunity to study several cognitive functions in a causal manner [21], [29]. TMS has been combined with several functional imaging techniques: positron emission tomography (PET) [18], single photon emission computed tomography (SPECT) [16], and functional magnetic resonance imaging (fMRI) [3]. Nonetheless, TMS interleaved with EEG provides, together with high temporal resolution, unique information about the characteristics of cortical reactivity and connectivity [8], [9].

A technical drawback of EEG/TMS coregistrations is that the TMS impulse induces high amplitude and long-lasting artifacts on the EEG trace (see Fig. 1). The term "artifact" denotes any potential, not generated by the brain, that affects the EEG signals and thus modifies, twists, or deletes the normal cerebral electric signal. The magnetic field generated by TMS usually saturates the EEG electrodes for 20-30 ms and even with special amplifiers designed to work with MRI, the electrodes cannot correctly record the cerebral activity in this period of time.

To solve this problem two coarse online strategies have been proposed in the literature. The first one uses sample-and-hold circuits to keep constant the output of the amplifiers during the impulse [8]. The amplifiers return to their normal operation after the impulse. The second method turns off the amplifiers $10 \mathrm{~ms}$ 
after the impulse. Besides the high costs due to the complex circuits, these methods cut off the EEG trace during TMS stimulation and therefore the information of the signal around the impulse is irremediably lost [17], [20].

An alternative to online strategies is represented by offline approaches. In this case, the artifacts are removed only after the complete acquisition of the EEG/TMS coregistration. Offline artifact suppression strategies are still in their infancy. In [4] and [28], the authors propose to remove TMS-induced artifacts by simply subtracting the mean artifact. This strategy is indeed effective to remove physiological artifacts but residual electrical artifacts are not expected to be completely eliminated. In [1] and [19], the authors use least mean square algorithms to remove fMRI-induced artifacts from EEG signals. Nonetheless TMS-induced artifacts are more energetic and less uniform than fMRI-induced artifacts and therefore poor performances are expected if these algorithms are applied to TMS.

In this brief, we propose an offline Kalman filter approach to remove TMS-induced artifacts from EEG recordings. The Kalman filter is applied to the linear system arising from the combination of the dynamic models, identified from data, describing EEG and TMS signals generation. The keystone of the approach is the use of time-varying covariance matrices suitably tuned on the physical parameters of the problem that allow one to model the non-stationary components of the EEG/TMS signal (neglected by conventional stationary filtering). The approach guarantees an efficient deletion of TMS-induced artifacts while preserving the integrity of EEG signals around TMS impulses. Experimental results show that the proposed strategy achieves a significant performance improvement over the existing offline strategies (Wiener filter). A preliminary version of this brief appeared in [14].

The rest of this brief is organized as follows. In Section II, some basic data structures are defined and the preprocessing phase is briefly motivated. In Section III, the models for EEG and TMS are introduced and the Kalman filter is designed. In Section IV, real data experiments prove the effectiveness of the proposed approach. In Section V, the main contributions of this brief are summarized and future research directions are highlighted.

Notation: The following notation will be used in the brief:

$\mathbb{R}^{+}$is the set of all positive real numbers. Bold lower case and capital letters stand for real column vectors and real matrices, respectively. $\operatorname{diag}\{\mathbf{A}, \mathbf{B}\}$ denotes a block diagonal matrix with square matrices $\mathbf{A}$ and $\mathbf{B}$ on the diagonal. $\mathbf{0}_{m \times n}$ and $\mathbf{I}_{n}$ denote the $m \times n$ matrix of zeros and the identity matrix of order $n$.

\section{PRELiminaries}

Through this brief, we will refer to the following data structures. The basic structural unit is the record. A record corresponds to some tens of seconds EEG/TMS coregistration on multiple channels and it is divided into $M$ data sets of $S$ samples. Each data set is supposed to contain a single TMS-impulse or stimulus having regular time spacing.

Fig. 2 shows a typical data set of 750 samples. Up to sample 510 the EEG/TMS trace includes only the EEG signal. At sample 510, a TMS-impulse induces an artifact on the trace. The duration of the impulse is about 30 samples.

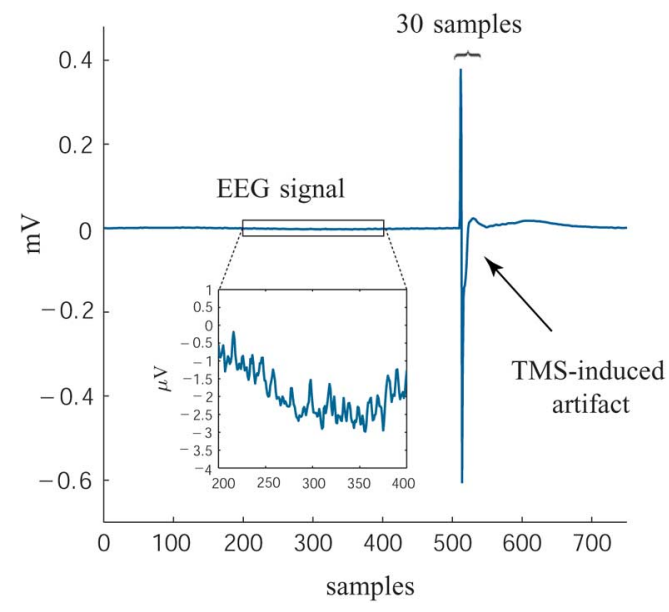

Fig. 2. Data set containing a TMS-induced artifact.

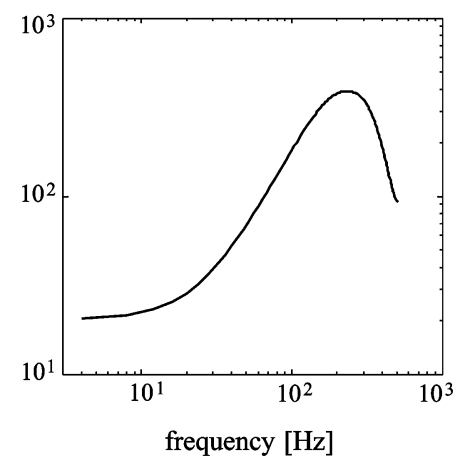

Fig. 3. Spectrum of a pure TMS-induced artifact recorded on a manikin.

Note that in spite of the electric shielding of EEG electrodes, power lines usually induce sinusoidal oscillations on the EEG signal due to the $50 \mathrm{~Hz}$ carrier. In order to avoid distorted results, these oscillations must be removed (e.g., by using standard Fourier series techniques) in a preprocessing phase preceding the application of the Kalman filter. Notice that since scarce EEG activity is present at $50 \mathrm{~Hz}$, the carrier can be removed without significant loss of information. Hereafter, $s(t)$ will denote the signal including the TMS-induced artifact and the EEG signal, with the 50-Hz carrier suppressed.

\section{KALMAN FILTER DESIGN}

The available signal $s(t)$ results from the combination of an electroencephalographic signal $\operatorname{eeg}(t)$, representing the natural electric activity of the brain and of a high amplitude signal $\operatorname{tms}(t)$, representing the artifact induced by the magnetic stimulation. Obviously $\operatorname{tms}(t)$ is present only in correspondence of a stimulus. The signals relative to the $i$ th data set of a record will be denoted $s_{i}(t), \operatorname{eeg}_{i}(t)$, and $\operatorname{tms}_{i}(t), i=1,2, \ldots, M$. Signal $\operatorname{eeg}_{i}(t)$ can be considered as a stationary zero mean signal [10]. On the other hand, $\operatorname{tms}_{i}(t)$, for its impulsive nature, is a non-stationary signal.

A pure TMS-induced artifact can be achieved by stimulating the head of a manikin. Analyzing the spectrum of this artifact (see Fig. 3), we observe that although most of signal energy is concentrated at high frequencies $(300 \mathrm{~Hz})$, several components are present at low frequencies (around $10 \mathrm{~Hz}$ ). Therefore, since the EEG signals usually range from 1 to $70 \mathrm{~Hz}$, trivial low-pass, 
high-pass filters cannot be exploited to separate EEG signals from the magnetic artifacts, since significant components of the EEG signal would be cut off.

Let us make the following assumptions:

1) $s_{i}(t)=\operatorname{eeg}_{i}(t)+\operatorname{tms}_{i}(t)$;

2) $\operatorname{eeg}_{i}(t)$ and $\mathrm{tms}_{i}(t), \forall t$, are uncorrelated.

Note that 1) and 2) are simplifying assumptions which most likely do not strictly hold in practice. Nevertheless, they allow us to develop a filtering procedure that will prove to be effective in removing TMS artifacts from EEG recordings.

According to the first hypothesis, in the record we have

$$
\begin{aligned}
& \frac{1}{M} \sum_{i=1}^{M} s_{i}(t+(i-1) S)= \\
& \frac{1}{M}\left(\sum_{i=1}^{M} \operatorname{eeg}_{i}(t+(i-1) S)+\sum_{i=1}^{M} \operatorname{tms}_{i}(t+(i-1) S)\right) .
\end{aligned}
$$

Since eeg $g_{i}(t)$ is supposed to be a zero mean signal

$$
\frac{1}{M} \sum_{i=1}^{M} \operatorname{eeg}_{i}(t+(i-1) S) \simeq 0
$$

and (1) approximately equals $\overline{\operatorname{tms}}(t)$, the mean artifact. If the artifacts were purely deterministic, then $\operatorname{tms}_{i}(t)=\overline{\operatorname{tms}}(t)$ for all $i$, and they could be removed by the simple subtraction

$$
\widehat{\operatorname{eeg}}_{i}(t)=s_{i}(t)-\overline{\operatorname{tms}}(t)
$$

where $\widehat{\mathrm{eeg}}_{i}(t)$ is the EEG signal without the artifact. Nevertheless, TMS-induced artifacts are not purely deterministic and, therefore, (2) is not sufficient to remove the magnetic artifact from $s_{i}(t)$. This drove us to devise an alternative and more effective strategy.

The Kalman filter (see, e.g., [12]) turned out to be an appealing solution. To design this filter a state-space model describing the signals generation mechanism is necessary, therefore two dynamic systems, modelling EEG and TMS signals generation, need to be determined. For the sake of simplicity, hereafter, the subscript " $i$ " (that refers to the data set) will be dropped.

According to the existing literature [10], for the EEG signal, autoregressive (AR) models have been considered

$$
\operatorname{eeg}(t)=\frac{1}{1+a_{1} q^{-1}+\cdots+a_{N_{a}} q^{-N_{a}}} e_{E}(t)
$$

i.e., signal eeg $(t)$ is supposed to be generated by a white noise $e_{E}(t)$ filtered by a function $1 / A(q)$, where $A(q)=1+a_{1} q^{-1}+$ $\cdots+a_{N_{a}} q^{-N_{a}}$ is a polynomial in the shift operator $q$, such that $q^{-1} x(t)=x(t-1)$. For the AR model order selection, several works can be found in the literature (see, e.g., [11] and [26]).

Differently from the EEG, the determination of a model for TMS has not been deeply investigated in the literature. Let us first model the deterministic part $\overline{t m s}(t)$. Due to the impulsive nature of the TMS artifacts, output error (OE) models with impulsive inputs can be considered

$$
\overline{\operatorname{tms}}(t)=\frac{B(q)}{F(q)} u(t-1)+v(t)
$$

where $u(t)$ is a unitary impulse that assumes the value 1 in correspondence of a stimulus and $v(t)$ represents the stochastic part of the OE model.

The previous AR and OE models can be utilized to build a linear state-space system describing the overall EEG/TMS signal. Consider the following state-space realization of model (3)

$$
\left\{\begin{array}{l}
\mathbf{x}_{E}(t+1)=\mathbf{A}_{E} \mathbf{x}_{E}(t)+\mathbf{G}_{E} e_{E}(t) \\
\operatorname{eeg}(t)=\mathbf{C}_{E} \mathbf{x}_{E}(t)
\end{array}\right.
$$

where

$$
\begin{aligned}
\mathbf{A}_{E} & =\left[\begin{array}{ccccc}
-a_{1} & -a_{2} & \cdots & -a_{N_{a}-1} & -a_{N_{a}} \\
1 & 0 & \cdots & 0 & 0 \\
0 & 1 & \cdots & 0 & 0 \\
\vdots & \vdots & \ddots & \vdots & \vdots \\
0 & 0 & \cdots & 1 & 0
\end{array}\right] \\
\mathbf{G}_{E} & =\left[\begin{array}{lllll}
1 & 0 & \cdots & 0 & 0
\end{array}\right]^{T} \\
\mathbf{C}_{E} & =\left[\begin{array}{lllll}
1 & 0 & \cdots & 0 & 0
\end{array}\right]
\end{aligned}
$$

$\mathbf{x}_{E}(t) \in \mathbb{R}^{N_{a}}$ is the EEG state vector and $e_{E}(t)$ is a zero-mean white noise of variance $\sigma_{F}^{2}$. Analogously, consider the following state-space realization of model (4):

$$
\left\{\begin{array}{l}
\mathbf{x}_{T}(t+1)=\mathbf{A}_{T} \mathbf{x}_{T}(t)+\mathbf{B}_{T} u(t) \\
\overline{\operatorname{tms}}(t)=\mathbf{C}_{T} \mathbf{x}_{T}(t)+v(t)
\end{array}\right.
$$

where $\mathbf{x}_{T}(t) \in \mathbb{R}^{\rho}$ is the TMS state vector, $\left\{\mathbf{A}_{T}, \mathbf{B}_{T}, \mathbf{C}_{T}\right\}$ with $\mathbf{A}_{T} \in \mathbb{R}^{\rho \times \rho}, \mathbf{B}_{T} \in \mathbb{R}^{\rho \times 1}, \mathbf{C}_{T} \in \mathbb{R}^{1 \times \rho}$ is the control canonical form of (4), $\rho=\max \{\operatorname{deg}(B(q)), \operatorname{deg}(F(q))\}$ and $v(t)$ is a zero-mean white noise of variance $\sigma_{v}^{2}$. The variances $\sigma_{E}^{2}$ and $\sigma_{v}^{2}$ of $e_{E}(t)$ and $v(t)$ required for Kalman filter design, are determined from the identification of the EEG and TMS models, respectively.

Collecting systems (5) and (6) together, we obtain the extended dynamic system

$$
\left\{\begin{array}{l}
\mathbf{x}(t+1)=\mathbf{A} \mathbf{x}(t)+\mathbf{B} u(t)+\mathbf{G} e_{E}(t) \\
\operatorname{eeg}(t)+\overline{\operatorname{tms}}(t)=\mathbf{C} \mathbf{x}(t)+v(t)
\end{array}\right.
$$

where

$$
\begin{aligned}
\mathbf{A} & =\operatorname{diag}\left\{\mathbf{A}_{E}, \mathbf{A}_{T}\right\} \\
\mathbf{B} & =\left[\begin{array}{c}
\mathbf{0}_{N_{a} \times 1} \\
\mathbf{B}_{T}
\end{array}\right] \\
\mathbf{G} & =\left[\begin{array}{c}
\mathbf{G}_{E} \\
\mathbf{0}_{\rho \times 1}
\end{array}\right] \\
\mathbf{C} & =\left[\begin{array}{ll}
\mathbf{C}_{E} & \mathbf{C}_{T}
\end{array}\right] \\
\mathbf{x}(t) & =\left[\begin{array}{ll}
\mathbf{x}_{E}(t)^{T} & \mathbf{x}_{T}(t)^{T}
\end{array}\right]^{T} .
\end{aligned}
$$

Note that (7) only models the stationary part of signal $s(t)$. To take into account the non-stationarities of $s(t)$ we carry out two 
changes in model (7). Actually, the non-stationary components of signal $s(t)$ can be effectively modeled using time-variant covariance matrices suitably tuned on the physical parameters of the problem.

First of all, we introduce a stochastic component into the TMS model as an additive disturbance vector $\mathbf{e}_{T}(t)$ in the dynamic equation of (6). Process $\mathbf{e}_{T}(t)$ is a non-stationary white noise modeling the variance of the TMS artifact during the first peak of the stimulus. System (7) is thus modified as follows:

$$
\left\{\begin{array}{l}
\mathbf{x}(t+1)=\mathbf{A} \mathbf{x}(t)+\mathbf{B} u(t)+\underbrace{\left[\begin{array}{cc}
\mathbf{G}_{E} & \mathbf{0}_{N_{a} \times \rho} \\
\mathbf{0}_{\rho \times 1} & \mathbf{I}_{\rho}
\end{array}\right]}_{\mathbf{G}_{M}} \mathbf{e}(t) \\
\operatorname{eeg}(t)+\overline{\operatorname{tms}}(t)=\mathbf{C} \mathbf{x}(t)+v(t)
\end{array}\right.
$$

where $\mathbf{e}(t)=\left[e_{E}(t) \mathbf{e}_{T}(t)^{T}\right]^{T}$

$$
\operatorname{var}\left\{\mathbf{e}_{T}(t)\right\}= \begin{cases}\sigma_{T}^{2} \mathbf{I}_{\rho}, & \text { if } t_{s} \leq t \leq t_{s}+d \\ \mathbf{0}_{\rho \times \rho}, & \text { otherwise. }\end{cases}
$$

$t_{s}$ denotes the sample at which the stimulus is generated while $d$ is the number of samples of the first peak of the TMS. The variance $\sigma_{T}^{2}$ is a tuning parameter of the Kalman filter. To summarize, the complete covariance matrix $\mathbf{Q}(t)=\operatorname{var}\{\mathbf{e}(t)\}$ is given by

$$
\mathbf{Q}(t)= \begin{cases}\operatorname{diag}\left\{\sigma_{E}^{2}, \sigma_{T}^{2} \mathbf{I}_{\rho}\right\}, & \text { if } t_{s} \leq t \leq t_{s}+d \\ \operatorname{diag}\left\{\sigma_{E}^{2}, \mathbf{0}_{\rho \times \rho}\right\}, & \text { otherwise. }\end{cases}
$$

Second, we replace $v(t)$ by a noise $\eta(t)$ whose variance changes in time according to

$$
R(t)=\operatorname{var}\{\eta(t)\}= \begin{cases}g(t), & \text { if } t_{s} \leq t \leq t_{s}+d_{\text {tot }} \\ 0, & \text { otherwise }\end{cases}
$$

where

$$
g(t)= \begin{cases}\sigma_{v}^{2}, & \text { if } t_{s} \leq t \leq t_{s}+d \\ \sigma_{v}^{2} e^{-\alpha\left(t-t_{s}-d\right)}, & \text { if } t_{s}+d<t \leq t_{s}+d_{\text {tot }} .\end{cases}
$$

The idea behind this choice is that the effect of the TMS on the EEG tends to decrease after the first peak of the TMS stimulus. Such an effect has been modeled by an exponential decay. The tuning parameter $\alpha \in \mathbb{R}^{+}$modulates the decay rate of the exponential while $d_{\text {tot }}$ is the expected length (expressed in number of samples) of the TMS effect on the EEG trace.

In order to recover the EEG signal without the artifact, the Kalman filter is applied to system (8), with the measurement noise $v(t)$ replaced by $\eta(t)$

$$
\begin{aligned}
\widehat{\mathbf{x}}(t+1 \mid t) & =\mathbf{A} \widehat{\mathbf{x}}(t \mid t)+\mathbf{B} u(t) \\
\mathbf{P}(t+1 \mid t) & =\mathbf{A} \mathbf{P}(t \mid t) \mathbf{A}^{T}+\mathbf{G}_{M} \mathbf{Q}(t+1) \mathbf{G}_{M}^{T} \\
\widehat{\mathbf{x}}(t+1 \mid t+1) & =\widehat{\mathbf{x}}(t+1 \mid t)+\mathbf{K}(t+1) \xi(t+1) \\
\mathbf{P}(t+1 \mid t+1) & =\mathbf{P}(t+1 \mid t)-\mathbf{K}(t+1) \mathbf{C P}(t+1 \mid t)
\end{aligned}
$$

where

$$
\begin{aligned}
\xi(t+1) & =\operatorname{eeg}(t+1)+\overline{\operatorname{tms}}(t+1)-\mathbf{C} \widehat{\mathbf{x}}(t+1 \mid t) \\
\mathbf{K}(t+1) & =\mathbf{P}(t+1 \mid t) \mathbf{C}^{T}\left[\mathbf{C P}(t+1 \mid t) \mathbf{C}^{T}+R(t+1)\right]^{-1} .
\end{aligned}
$$

At each time $t$, the Kalman filter delivers an estimate $\widehat{\mathbf{x}}(t \mid t)=$ $\left[\widehat{\mathbf{x}}_{E}(t \mid t)^{T} \widehat{\mathbf{x}}_{T}(t \mid t)^{T}\right]^{T}$ of the state $\mathbf{x}(t)$. Hence, the sought estimate of the EEG signal is given by

$$
\widehat{\operatorname{eeg}}(t)=\mathbf{C}_{E} \widehat{\mathbf{x}}_{E}(t \mid t), \quad \text { for all } t=1,2, \ldots, S \text {. }
$$

\section{EXPERIMENTAL RESULTS}

\section{A. Database, Subjects, and Experimental Equipment}

The database we used to test the effectiveness of the proposed approach consists of seven records. A single record corresponds approximately to a 22 -s acquisition on 32 channels. Each record is divided into 15 data sets of 1500 samples (the sampling time is $0.98 \mathrm{~ms}$ ). Each data set contains a single TMS impulse. The database was acquired at Dipartimento di Neuroscience, Sezione di Neurologia, Università di Siena. Experiments were carried out on 10 healthy volunteers (seven males) aged 22-43 years (average 28.5 years). All subjects gave their written informed consent for the study which was approved by the local ethical committee. The experiment was well tolerated by all subjects.

Let us now turn the attention to the experimental equipment. Single-pulse TMS was generated using Magstim Super Rapid biphasic stimulator and delivered through commercially available eight-shaped or circular coils (frequencies: 0.1 and $10 \mathrm{~Hz}$ ). The specifications of the equipment are: maximum discharge voltage $2 \mathrm{kV}$, maximum discharge current $7 \mathrm{kA}$, maximum magnetic field $2 \mathrm{~T}$, rising time of the magnetic field $60 \mu \mathrm{s}$, length of the impulse $250 \mu \mathrm{s}$.

EEG recording was carried out with Micromed equipment. To assure a good temporal precision we set the sampling frequency to $1024 \mathrm{~Hz}$. The amplifier was optically connected to a PC running the Brain-Quick System Plus package and to a shielded cap containing the electrodes. The cap records 32 channels: 30 channels are dedicated to the electrodes disposed according to the international "10-20 EEG System," while the last two channels are used for the electromyography of hand muscles. Before starting the EEG/TMS coregistration, the TMS threshold of each subject was determined according to standardized criteria [22]. The intensity of TMS pulses was set to $90 \%$, $110 \%$, and $120 \%$ of the individual motor threshold. The scalp region stimulated by TMS corresponded to the position of $\mathrm{C} 3$ electrode, according to the standard nomenclature reported in [24].

\section{B. Identification of EEG and TMS Models}

For the sake of exposition, the experimental results presented hereafter refer to one data set of a single subject. The performance of the proposed procedure does not change significantly for different data sets, records, and subjects.

For the EEG signal, AR models of different orders have been estimated and compared using the MATLAB System Identification Toolbox, according to Fit (percentage of the signal correctly reproduced by the model) and residuals autocorrelation. For the identification procedure, we used 2000 samples not affected by magnetic artifacts on a single channel. An AR(3) model, with $A(q)=1-1.354 q^{-1}+0.6846 q^{-2}-0.3036 q^{-3}$ was selected 


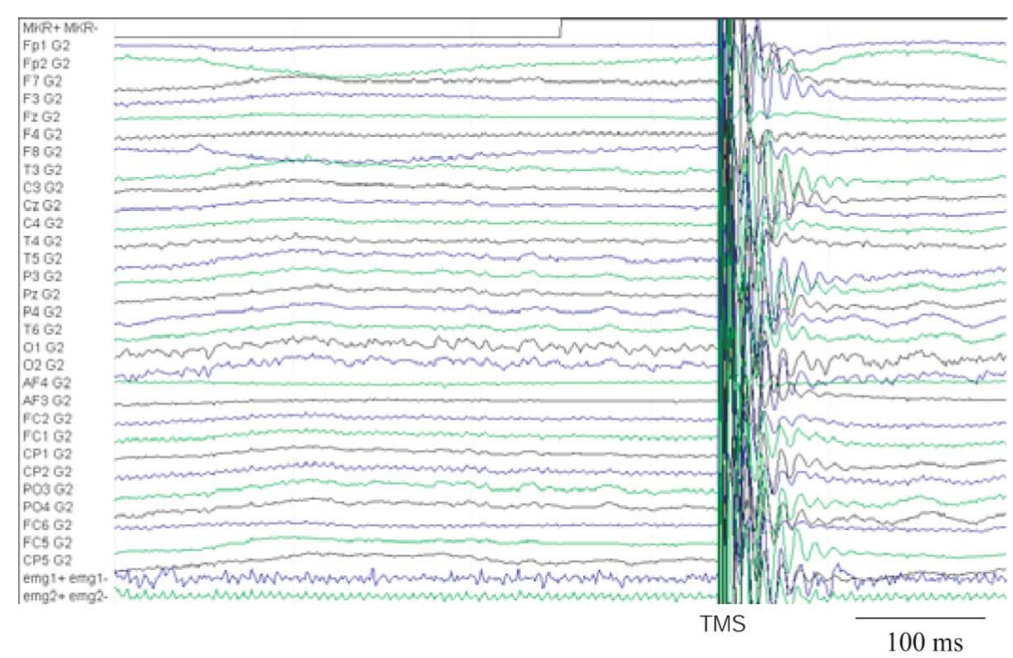

(a)

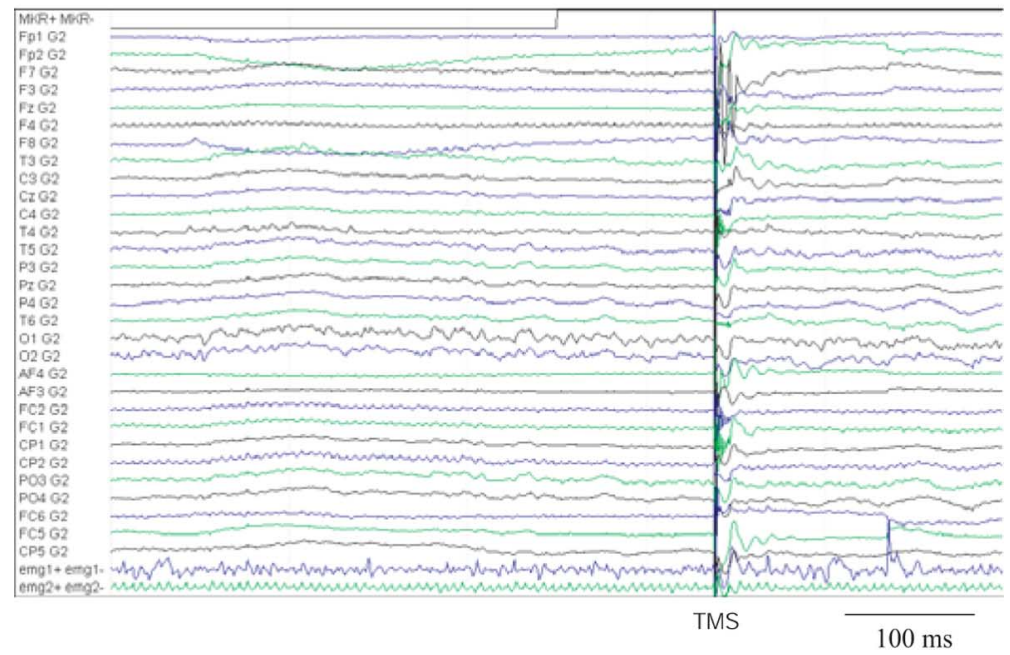

(b)

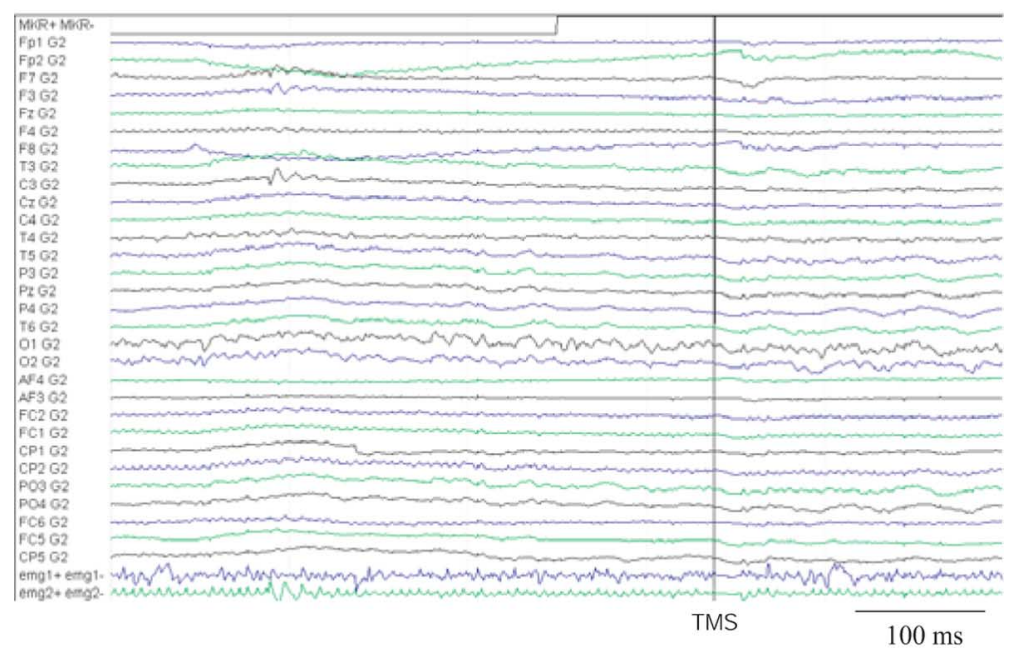

(c)

Fig. 4. (a) EEG/TMS trace on the 32 channels before the filtering. The signals after the application of: (b) a Wiener filter of order 15 and (c) the Kalman filter. In correspondence of the black vertical line, a TMS impulse is generated.

for the considered data set. To test the validity of this model we carried out validation tests using data from different channels: the considered AR(3) turned out to be a reliable model for all the channels. Higher order AR models have been also employed in the TMS removal procedure, but they did not provide a significant improvement of the results.

For the TMS, OE models of different orders were identified using five samples before and 35 samples after the stimulus. The 


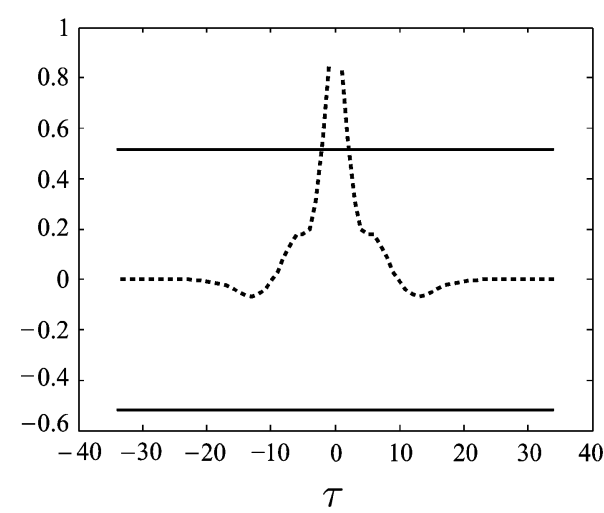

(a)

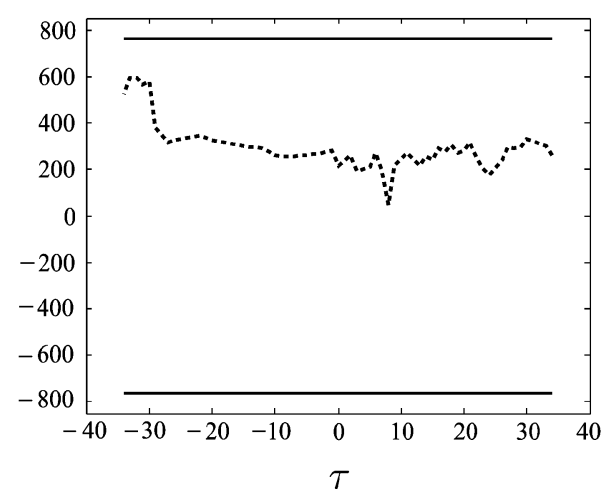

(b)

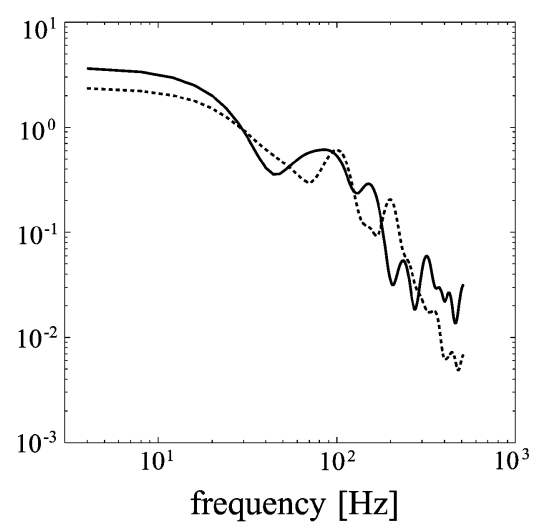

(c)

Fig. 5. Validation of the Kalman filter. (a) $\hat{R}_{\xi}^{N}(\tau) / \hat{R}_{\xi}^{N}(0)$ and the $99 \%$ confidence region $(N=1500, H=35)$; (b) $\hat{R}_{\xi}^{N} \widehat{\text { eeg }}(\tau)$ and the $99 \%$ confidence region ( $N=1500, H=35)$; (c) Spectrum of $\widehat{\operatorname{eeg}}(t)$ (solid line) and eeg $(t)$ (dashed line). $\widehat{e e g}(t)$ has been computed using 80 samples (including the artifact) on a single channel.

validation procedure was conducted on 15 stimuli. The $\mathrm{OE}(3,3)$ proved to be the best choice for TMS, suitably trading off accuracy of measured output reproduction and simplicity of model structure.

When validating the model using signals from other channels, we observed that differently from EEG, TMS model has only local validity (in fact in general, TMS artifacts are higher in the electrodes close to the stimulation site). However, repeating the identification procedure in the other channels, the $\mathrm{OE}(3,3)$ model still revealed the most suited structure.

\section{Tuning of the Kalman Filter and Discussion of the Results}

From the analysis of the data, we observed that the number of samples $d$ of the first peak of the TMS impulse is approximately equal to 4 and the effect of TMS on the EEG trace is about 30 samples long. Hence, we set $d_{\text {tot }}=30$. Moreover, after some trials, we chose $\sigma_{T}^{2}=0.1$ and $\alpha=0.3$. Note that the previous parameters are equal for all the 32 channels. The Kalman filter was initialized with

$$
\hat{\mathbf{x}}(0 \mid-1)=\mathbf{0}_{6 \times 1}, \quad \mathbf{P}(0 \mid-1)=\left[\begin{array}{cc}
\mathbf{I}_{3} & \mathbf{0}_{3 \times 3} \\
\mathbf{0}_{3} & 10^{-6} \mathbf{I}_{3}
\end{array}\right] .
$$

The EEG/TMS signals in our database were processed using the Kalman filter and a stationary filter, the Wiener filter, that has been used as a benchmark. Fig. 4(a) shows the EEG/TMS signals in a reference data set before the filtering. Fig. 4(b) shows the same signals after the application of a Wiener filter of order 15. In spite of an amplitude reduction, the artifacts have not been removed. Similar results were obtained with filters of different order. A basic condition for the application of the Wiener filter is that $\operatorname{tms}(t)$ can be modeled as a stationary signal. But since tms $(t)$ is indeed non-stationary, a satisfactory deletion of TMS-induced artifacts can not be expected. Fig. 4(c) shows the EEG/TMS signals after the application of the Kalman filter. In this case, the effect of the magnetic artifacts on the EEG recordings has been strongly reduced, while preserving the integrity of EEG signals around TMS impulses.

For a further validation of the proposed Kalman filter approach, some standard tests have been considered. First, statistical tests on the Kalman filter residuals $\xi(t)$ have been performed. It has been observed that $\hat{R}_{\xi}^{N}(\tau) / \hat{R}_{\xi}^{N}(0)$, $\tau=0,1, \ldots, H$, where $\hat{R}_{\xi}^{N}(\tau)$ is the sample autocorrelation of the residuals computed on $N$ samples, is always inside the 99\% confidence region, except for $\tau=1$ [see Fig. 5(a)]. It has also been noticed that $\hat{R}_{\xi, \widehat{e e g}}^{N}(\tau)$ (the sample cross-correlation between $\xi(t)$ and $\widehat{\operatorname{eeg}}(t)$ ) keeps always inside the $99 \%$ confidence region, thus showing that there is no evidence in the data that $\xi(t)$ and $\widehat{e e g}(t)$ are correlated [see Fig. 5(b)].

Second, a frequency analysis has been conducted. The spectra of eeg $(t)$ and eeg $(t)$ proved to be similar for each channel [see Fig. 5(c)]. Therefore, we can infer that $\widehat{\operatorname{eeg}}(t)$ has the characteristics of an electroencephalographic signal.

The proposed Kalman filter approach proved to be computationally efficient. Processing the EEG/TMS trace reported in Fig. 5(a) takes about $32 \mathrm{~s}$ on a laptop equipped with Pentium IV, $3 \mathrm{GHz}$ processor and $512 \mathrm{MB}$ RAM. However, if the code is translated from MATLAB to a lower level programming language, we are allowed to expect a $30 \%$ computation time reduction.

\section{CONCLUSION AND FUtURE WORK}

In this brief, an offline Kalman filter approach to remove TMS-induced artifacts from EEG recordings is proposed. Current results show that an effective procedure to screen out the artifacts is the following. First, identify from data two models describing EEG and TMS signals generation and collect them in an extended state-space system. According to the existing literature, we chose an AR model for EEG. An OE model for TMS is proposed in this brief. Second, apply the Kalman filter, with time-variant covariance matrices suitably tuned on real data, to the extended system. Such peculiar covariance matrices allow us to model the non-stationary components of the EEG/TMS signal, neglected by conventional offline filtering approaches.

The Kalman filter has been tailored to the specific application of TMS-induced artifacts removal, by suitably tuning the covariance matrices $\mathbf{Q}$ and $R$ in order to cope with the impulsive nature of TMS artifacts. Therefore, other artifacts that can be encountered in the EEG/TMS research may not be suppressed by the proposed strategy. However, future developments may address the tuning of the Kalman filter on these artifacts as well. 
Although the obtained results are promising, still investigations are needed to validate the reliability of the estimated EEG signal. In particular, more accurate models of the interaction between TMS and EEG (e.g., with coupling nonlinear terms) should be addressed. Another objective of future research concerns an in-depth analysis of the stochastic component of the TMS, thus yielding more effective representations of the nonstationary behavior of the TMS signal. Finally, it would be interesting to analyze other estimation algorithms, like the Kalman smoother (see, e.g., [27]) or nonlinear non-stationary filters, in case of nonlinear models accounting for EEG/TMS joint dynamics.

\section{ACKNOWLEDGMENT}

The authors would like to thank L. Bechi, a graduate student with the Dipartimento di Ingegneria dell'Informazione, Università di Siena, for his support during the preparation of this brief.

\section{REFERENCES}

[1] P. J. Allen, O. Josephs, and R. Turner, "A method for removing imaging artifact from continuous EEG recorded during functional MRI," Neuroimage, vol. 12, no. 2, pp. 230-239, 2000.

[2] F. Bartoli and S. Cerutti, "An optimal linear filter for the reduction of noise superimposed to the EEG signal," J. Biomed. Eng., vol. 5, no. 4, pp. 274-280, 1983.

[3] J. Baudewig, H. S. Siebner, S. Bestmann, F. Tergau, T. Tings, W. Paulus, and J. Frahm, "Functional MRI of cortical activations induced by transcranial magnetic stimulation (TMS)," NeuroReport, vol. 12, no. 16, pp. 3543-3548, 2001.

[4] G. Fuggetta, E. F. Pavone, V. Walsh, M. Kiss, and M. Eimer, "Corticocortical interactions in spatial attention: A combined ERP/TMS study," J. Neurophys., vol. 95, pp. 3277-3280, 2006.

[5] A. Galka, O. Yamashita, T. Ozaki, R. Biscay, and P. Valdés-Sosa, "A solution to the dynamical inverse problem of EEG generation using spatiotemporal Kalman filtering," Neurolmage, vol. 23, no. 2, pp. 435-453, 2004.

[6] S. D. Georgiadis, P. O. Ranta-aho, M. P. Tarvainen, and P. A. Karjalainen, "Single-trial dynamical estimation of event-related potentials: A Kalman filter-based approach," IEEE Trans. Biomed. Eng., vol. 52, no. 8, pp. 1397-1406, Aug. 2005.

[7] M. Hallett, "Transcranial magnetic stimulation and the human brain," Nature, vol. 406, no. 6792, pp. 147-150, 2000.

[8] R. J. Ilmoniemi, J. Virtanen, J. Ruohonen, J. Karhu, H. J. Aronen, R. Näätänen, and ,T. Katila, "Neuronal responses to magnetic stimulation reveal cortical reactivity and connectivity," NeuroReport, vol. 8, no. 16, pp. 3537-3540, 1997.

[9] K. Iramina, T. Maeno, Y. Kowatari, and S. Ueno, "Effects of transcranial magnetic stimulation on EEG activity," IEEE Trans. Magn., vol. 38, no. 5, pp. 3347-3349, Sep. 2002.

[10] B. H. Jansen, "Analysis of biomedical signals by means of linear modeling," Critical Rev. Biomed. Eng., vol. 12, no. 4, pp. 343-392, 1985.

[11] B. H. Jansen, J. R. Bourne, and J. W. Ward, "Autoregressive estimation of short segment spectra for computerized EEG analysis," IEEE Trans. Biomed. Eng., vol. 28, no. 9, pp. 630-638, Sep. 1981.

[12] E. W. Kamen and J. K. Su, Introduction to Optimal Estimation, 1st ed. New York: Springer, 1999.
[13] M. Kobayashi and A. Pascual-Leone, "Transcranial magnetic stimulation in neurology," Lancet Neurology, vol. 2, no. 3, pp. 145-156, 2003.

[14] F. Morbidi, A. Garulli, D. Prattichizzo, C. Rizzo, and S. Rossi, "A Kalman filter approach to remove TMS-induced artifacts from EEG recordings," in Proc. Eur. Control Conf., 2007, pp. 2201-2206.

[15] V. P. Oikonomou, A. T. Tzallas, and D. I. Fotiadis, "A Kalman filter based methodology for EEG spike enhancement," Comput. Methods Programs Biomed., vol. 85, no. 2, pp. 101-108, 2007.

[16] S. Okabe, R. Hanajima, T. Ohnishi, M. Nishikawa, E. Imabayashi, H. Takano, T. Kawachi, H. Matsuda, Y. Shiio, N. K. Iwata, T. Furubayashi, Y. Terao, and Y. Ugawa, "Functional connectivity revealed by single-photon emission computed tomography (SPECT) during repetitive transcranial magnetic stimulation (rTMS) of the motor cortex," Clinical Neurophys., vol. 114, no. 3, pp. 450-457, 2002.

[17] T. Paus, "Imaging the brain before, during, and after transcranial magnetic stimulation," Neuropsychologia, vol. 37, no. 2, pp. 219-224, 1999.

[18] R. Jech, C. J. Thompson, R. Comeau, T. Peters, and A. C. Evans, "Transcranial magnetic stimulation during positron emission tomography: A new method for studying connectivity of the human cerebral cortex," J. Neurosci., vol. 17, no. 9, pp. 3178-3184, 1997.

[19] S. Pellizzato, "Filtraggio adattativo di segnali EEG per la rimozione di artefatti durante acquisizione fMRI," Master's thesis, Dipartimento di Elettronica ed Informatica, Facoltà di Ingegneria, I, Università di Padova, Padova, Italy, 2003.

[20] G. W. Price, "EEG-dependent ERP recording: Using TMS to increase the incidence of a selected pre-stimulus pattern," Brain Res. Protocols, vol. 12, no. 3, pp. 144-151, 2004.

[21] S. Rossi and P. M. Rossini, "TMS in cognitive plasticity and the potential for rehabilitation," Trends Cognitive Sci., vol. 8, no. 6, pp. 273-279, 2004.

[22] P. M. Rossini, A. T. Barker, A. Berardelli, M. D. Caramia, G. Caruso, R. Q. Cracco, M. R. Dimitrijevic, M. Hallett, Y. Katayam, C. H. Lucking, A. L. Maertens de Noordhout, C. D. Marsden, N. M. F. Murray, J. Rothwell, M. Swash, and C. Tomberg, "Non-invasive electrical and magnetic stimulation of the brain, spinal cord and roots: Basic principles and procedures for routine clinical application: report of an IFCN commitee," Electroencephalogr. Clin. Neurophysiol., vol. 91, no. 2, pp. 79-92, 1994.

[23] P. M. Rossini and S. Rossi, "Transcranial magnetic stimulation: Diagnostic, therapeutic and research potential," Neurol., vol. 68, no. 7, pp. 484-488, 2007.

[24] F. Sharbrough, G. E. Chatrian, R. P. Lesser, H. Lüders, M. Nuwer, and T. W. Picton, "American electroencephalographic society guidelines for standard electrode position nomenclature," J. Clinical Neurophys., vol. 8, pp. 200-202, 1991.

[25] M. V. Spreckelsen and B. Bromm, "Estimation of single-evoked cerebral potentials by means of parametric modeling and Kalman filtering," IEEE Trans. Biomed. Eng., vol. 35, no. 9, pp. 691-700, Sep. 1988.

[26] H.-W. Steinberg, T. Gasser, and J. Franze, "Fitting autoregressive models to EEG time series: An empirical comparison of estimates of the order," IEEE Trans. Acoust., Speech, Signal Process., vol. ASSP-33, no. 1, pp. 143-150, Jan. 1985.

[27] M. P. Tarvainen, J. K. Hiltunen, P. O. Ranta-aho, and P. A. Karjalainen, "Estimation of nonstationary EEG with Kalman smoother approach: An application to event-related synchronization (ERS)," IEEE Trans. Biomed. Eng., vol. 51, no. 3, pp. 516-524, Mar. 2004.

[28] G. Thut, J. R. Ives, F. Kampmann, M. A. Pastor, and A. Pascual-Leone, "A new device and protocol for combining TMS and online recordings of EEG and evoked potentials," J. Neurosci. Methods, vol. 141, no. 2, pp. 207-217, 2005.

[29] V. Walsh and A. Cowey, "Transcranial magnetic stimulation and cognitive neuroscience," Nature Rev. Neurosci., vol. 1, no. 1, pp. 73-79, 2000. 